Supplement of Hydrol. Earth Syst. Sci., 22, 635-653, 2018

https://doi.org/10.5194/hess-22-635-2018-supplement

(C) Author(s) 2018. This work is distributed under

the Creative Commons Attribution 3.0 License.

(c) (1)
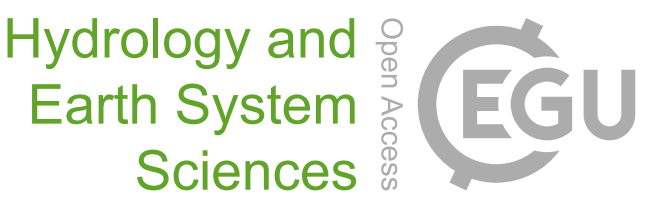

Supplement of

\title{
Mean transit times in headwater catchments: insights from the Otway Ranges, Australia
}

\section{William Howcroft et al.}

Correspondence to: William Howcroft (billhowcroft@gmail.com)

The copyright of individual parts of the supplement might differ from the CC BY 3.0 License. 


\begin{tabular}{|c|c|c|c|c|c|c|c|c|c|c|c|c|c|c|c|c|c|}
\hline Location $^{\mathrm{a}}$ & Date & $\begin{array}{r}Q \\
10^{3} \mathrm{~m}^{3} \\
\text { day }^{-1}\end{array}$ & $\begin{array}{r}\text { EC } \\
\mu \mathrm{S} / \mathrm{cm}\end{array}$ & $\begin{array}{l}\mathrm{pH} \\
\text { S.U. }\end{array}$ & $\begin{array}{r}\text { TDS } \\
\mathrm{mg} / \mathrm{L}\end{array}$ & $\begin{array}{l}\mathrm{HCO}_{3} \\
\mathrm{mg} / \mathrm{L}\end{array}$ & $\begin{array}{r}F \\
\mathrm{mg} / \mathrm{L}\end{array}$ & $\begin{array}{r}\mathrm{Cl} \\
\mathrm{mg} / \mathrm{L}\end{array}$ & $\begin{array}{r}\mathrm{Br} \\
\mathrm{mg} / \mathrm{L}\end{array}$ & $\begin{array}{r}\mathrm{NO}_{3} \\
\mathrm{mg} / \mathrm{L}\end{array}$ & $\begin{array}{r}\mathrm{SO}_{4} \\
\mathrm{mg} / \mathrm{L}\end{array}$ & $\begin{array}{r}\mathrm{Si} \\
\mathrm{mg} / \mathrm{L}\end{array}$ & $\begin{array}{r}\mathrm{Na} \\
\mathrm{mg} / \mathrm{L}\end{array}$ & $\begin{array}{r}\mathrm{Mg} \\
\mathrm{mg} / \mathrm{L}\end{array}$ & $\begin{array}{r}\mathrm{K} \\
\mathrm{mg} / \mathrm{L}\end{array}$ & $\begin{array}{r}\mathrm{Ca} \\
\mathrm{mg} / \mathrm{L}\end{array}$ & $\begin{array}{l}{ }^{3} \mathrm{H} \\
\mathrm{TU}\end{array}$ \\
\hline \multirow{4}{*}{$\begin{array}{l}\text { Upper } \\
\text { Lardners } \\
\text { (UL) }\end{array}$} & $10 / 07 / 2014$ & $\mathrm{~nm}^{\mathrm{c}}$ & 142 & 6.26 & 74 & 7.8 & 0.02 & 30.5 & 0.06 & 2.31 & 3.9 & 5.5 & 18.2 & 2.7 & 0.7 & 2.1 & $1.99 \pm 0.04^{b}$ \\
\hline & $28 / 09 / 2014$ & $\mathrm{~nm}$ & 168 & 6.03 & 83 & 15.1 & 0.03 & 30.1 & 0.07 & 0.68 & 3.4 & 6.7 & 18.8 & 3.4 & 1.3 & 3.1 & $1.77 \pm 0.04$ \\
\hline & $20 / 03 / 2015$ & $\mathrm{~nm}$ & 192 & 7.11 & 118 & 41.8 & 0.03 & 31.5 & 0.07 & 0.37 & 3.1 & 7.2 & 21.1 & 5.1 & 1.9 & 5.7 & $1.54 \pm 0.04$ \\
\hline & $10 / 09 / 2015$ & $\mathrm{~nm}$ & 154 & 6.48 & 81 & 11.3 & 0.03 & 33.0 & 0.08 & 1.72 & 4.2 & 6.1 & 18.7 & 2.8 & 1.1 & 2.4 & $1.99 \pm 0.03$ \\
\hline \multirow{5}{*}{$\begin{array}{l}\text { Lardners } \\
\text { Gauge } \\
\text { (LG) }\end{array}$} & $10 / 07 / 2014$ & 151.3 & 164 & 6.19 & 85 & 9.5 & 0.02 & 35.8 & 0.08 & 3.03 & 4.5 & 5.3 & 19.9 & 3.1 & 1.6 & 2.4 & $1.94 \pm 0.04$ \\
\hline & $28 / 09 / 2014$ & 32.8 & 182 & 6.45 & 94 & 18.3 & 0.02 & 34.0 & 0.09 & 1.21 & 3.3 & 6.2 & 22.6 & 3.8 & 1.3 & 3.4 & $1.94 \pm 0.04$ \\
\hline & 20/03/2015 & 5.0 & 197 & 6.71 & 114 & 35.0 & 0.03 & 35.3 & 0.10 & 0.29 & 2.7 & 6.6 & 21.7 & 4.9 & 1.9 & 5.0 & $1.64 \pm 0.04$ \\
\hline & $10 / 09 / 2015$ & 116.6 & 179 & 5.87 & 92 & 12.3 & 0.03 & 38.9 & 0.09 & 2.23 & 4.5 & 5.9 & 21.0 & 3.3 & 1.4 & 2.8 & $1.97 \pm 0.03$ \\
\hline & 4/11/2015 & 12.7 & 172 & 6.68 & 100 & 22.2 & 0.03 & 36.4 & 0.09 & 1.78 & 3.5 & 6.4 & 20.6 & 3.7 & 1.7 & 3.4 & $1.77 \pm 0.04$ \\
\hline \multirow{6}{*}{$\begin{array}{l}\text { Gellibrand } \\
\text { River @ } \\
\text { James } \\
\text { Access } \\
\text { (JA) }\end{array}$} & $13 / 03 / 2012^{d}$ & 18.5 & 160 & $\mathrm{~nm}$ & 93 & 39.0 & 0.07 & 26.0 & 0.05 & 0.05 & 3.3 & $\mathrm{~nm}$ & 14.7 & 3.0 & 1.5 & 5.0 & $1.90 \pm 0.09$ \\
\hline & $26 / 04 / 2012^{d}$ & 30.4 & 161 & $\mathrm{~nm}$ & 85 & 27.0 & 0.07 & 27.2 & 0.07 & 0.40 & 2.8 & $\mathrm{~nm}$ & 16.6 & 3.8 & 1.4 & 6.0 & $1.80 \pm 0.08$ \\
\hline & $10 / 07 / 2014$ & 255.2 & 151 & 6.70 & 74 & 10.0 & 0.03 & 27.6 & 0.06 & 2.41 & 3.6 & 6.2 & 17.6 & 2.6 & 1.0 & 2.6 & $2.04 \pm 0.04$ \\
\hline & $28 / 09 / 2014$ & 39.1 & 164 & 6.66 & 87 & 21.4 & 0.05 & 28.0 & 0.08 & 0.48 & 3.2 & 6.7 & 19.0 & 3.3 & 1.1 & 3.8 & $1.93 \pm 0.04$ \\
\hline & $20 / 03 / 2015$ & 8.8 & 162 & 6.71 & 94 & 28.5 & 0.03 & 28.0 & 0.07 & 0.08 & 2.9 & 6.3 & 18.6 & 3.6 & 1.5 & 4.4 & $1.73 \pm 0.04$ \\
\hline & $10 / 09 / 2015$ & 204.4 & 146 & 6.68 & 84 & 17.8 & 0.04 & 29.7 & 0.08 & 1.64 & 3.7 & 6.6 & 17.5 & 2.7 & 1.0 & 3.0 & $2.08 \pm 0.03$ \\
\hline \multirow{5}{*}{$\begin{array}{l}\text { Porcupine } \\
\text { Creek (PC) }\end{array}$} & $10 / 07 / 2014$ & 50.4 & 281 & 6.33 & 138 & 11.5 & 0.05 & 61.1 & 0.08 & 0.28 & 10.2 & 6.5 & 34.9 & 5.1 & 1.7 & 6.4 & $1.97 \pm 0.04$ \\
\hline & $27 / 09 / 2014$ & 3.3 & 380 & 6.76 & 200 & 51.2 & 0.04 & 68.4 & 0.17 & 0.21 & 5.1 & 7.2 & 44.6 & 7.2 & 2.2 & 14.0 & $1.68 \pm 0.04$ \\
\hline & $20 / 03 / 2015$ & 1.0 & 723 & 7.30 & 528 & 271.8 & 0.12 & 90.1 & 0.26 & 0.74 & 8.1 & 13.4 & 61.9 & 15.8 & 7.6 & 58.4 & $0.20 \pm 0.02$ \\
\hline & $10 / 09 / 2015$ & 9.7 & 319 & 6.51 & 171 & 15.0 & 0.04 & 83.1 & 0.15 & 0.31 & 9.1 & 7.2 & 41.3 & 6.1 & 1.7 & 6.8 & $2.08 \pm 0.04$ \\
\hline & $4 / 11 / 2015$ & 0.6 & 661 & 6.95 & 485 & 235.7 & 0.13 & 99.1 & 0.31 & 0.68 & 8.3 & 11.7 & 60.1 & 14.2 & 6.7 & 47.7 & $0.40 \pm 0.02$ \\
\hline \multirow{5}{*}{$\begin{array}{l}\text { Ten Mile } \\
\text { Creek (TC) }\end{array}$} & $10 / 07 / 2014$ & 8.6 & 397 & 6.43 & 184 & 7.8 & 0.05 & 90.4 & 0.22 & 0.54 & 13.5 & 4.6 & 54.3 & 7.1 & 1.8 & 3.5 & $1.74 \pm 0.04$ \\
\hline & $27 / 09 / 2014$ & 0.6 & 463 & 6.48 & 229 & 29.3 & 0.02 & 101.6 & 0.31 & 0.26 & 13.8 & 4.8 & 65.8 & 8.1 & 1.2 & 3.6 & $1.00 \pm 0.03$ \\
\hline & $20 / 03 / 2015$ & 0.2 & 406 & 6.47 & 191 & 4.6 & 0.02 & 102.8 & 0.33 & 0.08 & 10.0 & 4.8 & 59.1 & 5.8 & 0.9 & 2.3 & $0.44 \pm 0.02$ \\
\hline & $10 / 09 / 2015$ & 1.7 & 562 & 6.45 & 285 & 12.8 & 0.06 & 156.1 & 0.43 & 0.37 & 16.1 & 5.7 & 77.2 & 9.8 & 1.4 & 4.9 & $1.09 \pm 0.02$ \\
\hline & $4 / 11 / 2015$ & 0.1 & 392 & 6.49 & 199 & 7.6 & 0.03 & 107.8 & 0.34 & 0.22 & 11.8 & 4.6 & 57.6 & 5.8 & 1.0 & 2.5 & $0.53 \pm 0.02$ \\
\hline \multirow{2}{*}{$\begin{array}{l}\text { Yahoo } \\
\text { Creek (YC) }\end{array}$} & $11 / 07 / 2014$ & 23.0 & 368 & 5.73 & 221 & 37.8 & 0.03 & 94.1 & 0.20 & 0.24 & 13.7 & 5.7 & 53.3 & 7.9 & 2.1 & 6.1 & $2.14 \pm 0.04$ \\
\hline & $28 / 09 / 2014$ & 1.2 & 505 & 6.09 & 276 & 69.5 & 0.03 & 103.8 & 0.33 & 0.14 & 14.2 & 3.0 & 66.9 & 9.2 & 1.8 & 7.3 & $1.19 \pm 0.03$ \\
\hline
\end{tabular}




\begin{tabular}{|c|c|c|c|c|c|c|c|c|c|c|c|c|c|c|c|c|c|}
\hline & 20/03/2015 & 0.4 & 471 & 6.52 & 229 & 26.8 & 0.03 & 112.3 & 0.37 & 0.17 & 7.0 & 4.0 & 65.1 & 7.3 & 0.9 & 5.3 & $0.43 \pm 0.02$ \\
\hline & $10 / 09 / 2015$ & 3.9 & 569 & 6.22 & 283 & 5.6 & 0.04 & 153.0 & 0.37 & 0.41 & 24.1 & 5.2 & 72.8 & 10.9 & 2.0 & 8.7 & $1.30 \pm 0.03$ \\
\hline \multirow{5}{*}{$\begin{array}{l}\text { Love } \\
\text { Creek } \\
\text { Kawarren } \\
\text { (LK) }\end{array}$} & $10 / 07 / 2014$ & 102.9 & 402 & 6.66 & 197 & 21.7 & 0.13 & 83.8 & 0.18 & 1.28 & 14.5 & 5.0 & 47.2 & 7.6 & 5.2 & 10.2 & $1.85 \pm 0.03$ \\
\hline & $27 / 09 / 2014$ & 6.7 & 570 & 6.75 & 286 & 59.8 & 0.05 & 105.9 & 0.33 & 4.30 & 10.3 & 4.9 & 66.5 & 11.0 & 6.7 & 16.1 & $1.34 \pm 0.03$ \\
\hline & $20 / 03 / 2015$ & 2.0 & 527 & 6.99 & 286 & 62.0 & 0.04 & 114.3 & 0.34 & 0.94 & 9.1 & 6.1 & 66.2 & 8.9 & 3.4 & 15.2 & $0.48 \pm 0.02$ \\
\hline & $10 / 09 / 2015$ & 18.6 & 456 & 6.71 & 236 & 25.9 & 0.06 & 112.0 & 0.24 & 0.92 & 12.3 & 6.5 & 55.8 & 8.5 & 2.9 & 10.8 & $1.91 \pm 0.03$ \\
\hline & $4 / 11 / 2015$ & 1.2 & 522 & 6.23 & 307 & 68.8 & 0.07 & 124.0 & 0.37 & 1.02 & 10.9 & 5.8 & 65.6 & 9.6 & 2.7 & 17.9 & $0.58 \pm 0.02$ \\
\hline \multirow{4}{*}{$\begin{array}{l}\text { Love } \\
\text { Creek } \\
\text { Wonga } \\
\text { (LW) }\end{array}$} & $10 / 07 / 2014$ & 103.5 & 411 & 6.58 & 204 & 22.3 & 0.08 & 86.2 & 0.19 & 1.08 & 16.7 & 6.0 & 48.3 & 9.0 & 3.5 & 10.5 & $1.86 \pm 0.04$ \\
\hline & $28 / 09 / 2014$ & 6.0 & 721 & 6.93 & 367 & 84.2 & 0.10 & 135.9 & 0.42 & 3.33 & 11.8 & 6.4 & 76.7 & 17.8 & 6.4 & 23.5 & $1.34 \pm 0.03$ \\
\hline & $20 / 03 / 2015$ & 2.0 & 857 & 7.27 & 481 & 128.7 & 0.06 & 178.8 & 0.57 & 0.56 & 9.6 & 9.8 & 86.2 & 23.0 & 8.1 & 35.9 & $0.55 \pm 0.02$ \\
\hline & $10 / 09 / 2015$ & 19.6 & 514 & 6.82 & 271 & 37.3 & 0.07 & 125.2 & 0.26 & 1.15 & 13.8 & 7.0 & 57.7 & 11.1 & 3.5 & 14.1 & $1.88 \pm 0.03$ \\
\hline $\begin{array}{l}\text { Birnam } \\
\text { Rainfall }\end{array}$ & $27 / 09 / 2014$ & - & 80 & 6.49 & 25 & 5.7 & 0.00 & 8.5 & 0.02 & 0.23 & 1.5 & 0.0 & 5.1 & 0.7 & 2.6 & 0.8 & $2.45 \pm 0.04$ \\
\hline
\end{tabular}

Notes:
a) Locations on Fig. 1
b) Uncertainty
c) Not measured
d) Data from Atkinson (2014) 